\title{
Numerical modelling on stimulated Brillouin scattering characterization for Graphene-clad tapered silica fiber
}

\author{
Hui Jing Lee ${ }^{1, *}$, Fairuz Abdullah $^{1}$, and Aiman Ismail $^{1}$ \\ ${ }^{1}$ Center for Photonics Technologies, College of Engineering, Universiti Tenaga Nasional, Malaysia
}

\begin{abstract}
This paper presents finite numerical modelling on the cross-sectional region of tapered single mode fiber and graphene-clad tapered fiber. Surface acoustic wave propagation across the tapered surface region on tapered single mode fiber has a high threshold power at $61.87 \mathrm{~W}$ which is challenging to overcome by the incident pump wave. Surface acoustic wave propagation of fiber surface however made tapered wave plausible in the optical sensor application. This research introduces graphene as the cladding layer on tapered fiber, acoustic confinement occurs due to the graphene cladding which lowers the threshold power from 61.87 $\mathrm{W}$ to $2.17 \mathrm{~W}$.
\end{abstract}

\section{Introduction}

Stimulated Brillouin Scattering (SBS) is an optical scattering process caused by the non-linearity $\left(\chi^{3}\right)$ of a medium. The incident wave which is also known as the pump wave has to be higher than threshold power to generate acoustic wave [1]. The process of sound vibration fluctuates the refractive index of optical wave and scatters the injected pump wave. This effect creates a Stoke wave propagating in the opposite direction [2]. The scattering effect will transfer power from pump wave to Stoke wave therefore loss in energy is expected due to the fiber vibration. Because of this, acoustic frequency shift happens therefore lowers the frequency of Stoke wave from the original pump wave frequency. The Stoke wave will generate a strong optical Brillouin gain which can be used to amplify the weak injected wave [3].

Threshold power has both its functionality and limitation. In the communication field, SBS limitation could be signal loss, power saturation and backward propagation [4]. Hence, downside of Stoke wave is that it might saturates the amplifier and creates noise in the transmitter. Suppression of SBS can be done with several methods: one option would be to ensure the pump power is lower than the threshold power, the other option would be to increase the threshold power without dispersion penalty [5]. On the contrary, SBS with low threshold power has its functionality in lasers and amplifiers [6].

SBS has enormous potential in the application of sensing and optical communication. Low threshold power and short fiber length are two major indicators for a good sensor. In previous research, extensive experiments have been done to measure acoustic frequency shift across fibers. The focus is now on the threshold power therefore feasibility of different fibers in SBS applications are investigated [7]. Chalcogenide (ChG) fiber which secured acoustic confinement in the fiber core shows a low threshold power at $52 \mathrm{~mW}$ [8]. On the contrary, recent finding in SBS across tapered Silica fiber presented a high threshold power $(60 \mathrm{~W})$ which is undesired in SBS application [9]. This happens because Surface Acoustic Wave (SAW) at tapered waist region has minimum interaction with optical wave. Hence, gold and silver are two types of cladding materials proposed to overcome the high threshold power of tapered fiber.

A tapered single mode fiber (TSMF) is a heated and stretched standard single mode fiber which comprises of the non-tapered region, transition region and tapered waist region. An ideal TSMF diameter of tapered waist region around $0.8 \sim 3.0 \mu \mathrm{m}$ has smooth fiber surface and constant diameter [10-12]. TSMF has great plausibility in the sensor application. Light propagation across both adiabatic and non-adiabatic tapered fibers were both reported [13]. TSMF is also confirmed to have high sensitivity and is made possible to detect the change in protein concentration [14]. In addition, TSMF also has its functionality as a magnetic field sensor such that the magnetic fluid functions as the cladding layer for tapered fiber [15]. Also, functionality of TSMF was found in the refractive index based sensors and surface plasmon resonance (SPR) transducers $[16,17]$.

Studies shown that there are significant differences in the characteristics of tapered fiber clad with varied materials. One common similarity is that they are feasible in sensor application. For example, functionality of TSMF clad with Indium Tin Oxide (TIO) as a humidity sensor was shown [18]. Recent studies focus on graphene material which received great attention due to its uniqueness in optical, electrical, chemical and mechanical properties. The main focus would be on its optical properties with $97.7 \%$ of light transmittance rate [19]. Yao presented that graphene-clad microfiber enhances the evanescent field of fiber, it possesses ultrahigh nonlinearity therefore successfully generates Four Wave

\footnotetext{
* Corresponding author: LHjing@uniten.edu.my
} 
Mixing (FWM) which microfiber fails in such case [20]. Optical light was reported to confine at the graphene clad region, it is therefore applicable in the fiber optic nonlinear devices. Also, finding revealed uric acid detection using graphene-clad tapered fiber (GCTF) [21]. Functionality of GCTF in glucose detection was brought into discussion. Consequently, the functionality of graphene oxide clad fiber in aqueous ethanol sensing was proved [22]. Recent finding proved the functionality of graphene oxide clad tapered fiber bio-sensor for DNA detection [23]. Furthermore, tapered fiber partially covered with graphene layer is proved to enhance its optical conductivity [24].

Study regarding TSMF is scanty, realizing the gap in the extant literature, more research work is needed to investigate the potential of TSMF in SBS. However, so far none of the numerical solution tool has been used to model the exact SBS behaviour across TSMF or fiber with the variation of cladding materials [25]. The potential functionality of graphene-clad tapered fiber in SBS in yet to be studied. Hence, this paper performs numerical modelling using Finite Element Method (FEM) to study the stimulated Brillouin characterization on a grapheneclad tapered silica fiber.

FEM is a promising tool in computational Science and Engineering [26]. FEM has two approachable methods namely the Variational Method and Galerkin's Method. Generally, Galerkin's Method is preferable as it is more accessible for cases where Variational Method fails to handle [27]. Thorough literature reveals that FEM is a mature numerical modelling tool for fiber modelling such as for hollow fiber, inextensible fibers and ceramic matrix continuous [28-30].

\section{Stimulated Brillouin Scattering Formulation}

Fundamental of SBS characterization is based on the optical and acoustic wave interaction. When the incident pump wave exceeds the threshold power, acoustic wave will be generated. Fluctuation in the refractive index of optical wave will scatter the injected pump wave hence a Stoke wave propagates in the opposite direction occurs. Hence, due to the scattering effect, transfer power is transferred from the pump wave to Stoke wave. In SBS characterization, firstly the optical waveguide is solved then the acoustic waveguide is generated under a modematching condition. Based on the optical-acoustic interaction, the Brillouin gain and threshold power are calculated.

The fundamental optical mode property is solved as:

$$
\Delta_{t}^{2}+\left(\frac{2 \pi}{\lambda}\right)^{2}\left(n^{2}-n_{\text {eff }}^{2}\right) E=0
$$

where $\lambda$ is the wavelength of incidence light, $n$ and $n_{\text {eff }}$ are the refractive index and effective refractive index of fundamental optical wave, respectively. $E$ represents the optical spacial distribution whereas $\Delta_{t}$ signifies the transverse Laplacian operator in the reference of $\mathrm{x}$ and $\mathrm{y}$ plane [31].
Using $n_{e f f}$ and $\lambda$ from Equation (1), the optical wave propagation constant is solved [32]:

$$
\beta_{\text {optic }}=\frac{2 \pi n_{\text {eff }}}{\lambda}
$$

SBS characterization is performed under a modematching condition. The $\beta_{\text {optic }}$ in Equation 2 is then used to solve for the acoustic wave propagation by applying the Braggs Grating mathematical derivation to define the diffractive indices which refract and reflect at the interfaces:

$$
k_{\text {acoustic }}=2 \beta_{\text {optic }}
$$

This explains that the acoustic field is based on the $n_{\text {eff }}$ solved from Equation 1, the backward scattered wave explains the formation of Stoke wave [33]. Therefore, the acoustic velocity, $V_{a c}$ is hence defined as:

$$
V_{a c}=\frac{\omega}{k_{\text {acoustic }}}
$$

where $\omega$ is the angular energy. Hence, using the acoustic velocity, $V_{a c}$ solved from Equation (4), the Brillouin shift for acoustic wave at fundamental mode is solved [34]:

$$
v_{B}=\frac{2 n_{e f f} V_{a c}}{\lambda}
$$

The acoustic velocity, $V_{a c}$ is also used to solve for the acoustic frequency shift $v_{a c}$ :

$$
V_{a c}=v_{a c} \sqrt{\frac{1}{v_{1}^{2}}-\frac{1}{v_{2}^{2}}}
$$

where $v_{1}$ is the core velocity and $v_{2}$ is the clad velocity.

Formulation of Brillouin gain spectrum (BGS) is expressed as:

$$
g^{m}(v)=g_{p} \frac{\frac{\Delta v_{a c}(m)^{2}}{2}}{\left[v_{s}-\left(v_{p}-v_{a c}^{(m)}\right]\right]^{2}+\frac{\Delta v_{a c}(m)^{2}}{2}} \Gamma_{i j}
$$

where $v_{a c}^{(m)}$ is the m-th order acoustic frequency shift, $\Delta v_{a c}{ }^{(m)}$ represents the Full Width Half Maximum (FWHM) of the m-th order acoustic mode, $v_{p}$ is the pump frequency and $v_{s}$ is the stoke frequency. $\Gamma_{i j}$ signifies the overlapping factor between optical waveguide and acoustic waveguide:

$$
\Gamma_{i j}=\frac{\int\left[\left|H_{i}^{2}(x, y)\right|\left|U_{j}(x, y)\right|^{2}\right] d A}{\int\left|H_{i}(x, y)\right|^{4} d A \int\left|U_{j}(x, y)\right|^{2} d A}
$$

where $H_{i}(x, y)$ is the optical wave at fundamental mode and $U_{j}(x, y)$ is the acoustic wave displacement.

The fundamental mode of $v_{a c}$ is equivalent to $v_{B}$. The Brillouin peak gain is achieved under condition where the difference between pump and stoke frequency is equivalent to the Brillouin shift, $v_{B}[35,36]$.

$$
g_{B}(v)=g_{p}=\frac{4 \pi^{3} n_{e f}^{8} p_{12}^{2}}{c \lambda_{p}^{3} \rho_{o} v_{B} \Delta v_{B}}
$$


where $\rho_{12}$ signifies the elasto-optic coefficient, $\rho_{o}$ is the fiber core density, $c$ represents speed of light and $\Delta v_{B}$ is the FWHM of acoustic wave.

Substituting the Brillouin gain solve for the SBS threshold power:

$$
P_{t h}=\frac{\kappa A_{\mathrm{eff}}}{g_{p} L_{e f f} K}
$$

where $\kappa$ is the numerical factor manipulated by fiber length and is approximated to a constant value $\approx 21 . A_{\text {eff }}$ and $L_{e f f}$ are the effective area and effective length of fiber, respectively. $K$ is the polarization factor range between 1-2 [37].

\section{Results and Discussion}

This section firstly presents the fundamental optical and acoustic waveguides of tapered fiber (TF) and grapheneclad tapered fiber (GCTF) using FEM programmed in Fortran. The numerical output of the optic and acoustic are then used to calculate the Brillouin gain and threshold power parameters using Matlab. Design parameters for silica fiber and graphene are listed in Table $1[36,38]$.

Table 1. Design parameters for silica fiber and graphene

\begin{tabular}{|c|c|c|}
\hline Properties & Silica Fiber & Graphene \\
\hline$V_{\text {longitudinal }}(\mathrm{m} / \mathrm{s})$ & 5933 & 19,700 \\
\hline$V_{\text {shear }}(\mathrm{m} / \mathrm{s})$ & 3764 & 12,700 \\
\hline$\rho\left(\mathrm{kg} / \mathrm{m}^{3}\right)$ & 2202 & 1750 \\
\hline
\end{tabular}

\subsection{Optical wave}

FEM is used to solve for the optical wave propagation for TF and GCTF using Equation 1 and 2. The outer region for TF and GCTF are air and graphene, respectively. Table 2 lists the output parameters for the fundamental optic mode of TF and GCTF.

Table 2. Optical properties of TF and GCTF

\begin{tabular}{|c|c|c|}
\hline Properties & TF & GCTF \\
\hline$n_{\text {core }}$ & 1.4447 & 1.4447 \\
\hline$n_{\text {clad }}$ & 1 & 2 \\
\hline$n_{\text {eff }}$ & 1.1748 & 1.9327 \\
\hline$\beta_{\text {optic }}\left(\mu \mathrm{m}^{-1}\right)$ & 4.8621 & 8.2080 \\
\hline
\end{tabular}

Figure 1 (a), (b), and (c) show the fundamental Hy, Hx and $\mathrm{Hz}$ optical mode for TF with fiber radius $=0.5 \mu \mathrm{m}$. Total internal reflection is observed in Figure 1 (a) with the fundamental optic mode dominating in the y direction. The optic confinement is shown in Figure 1 (d) with peak intensity at 2.7 .

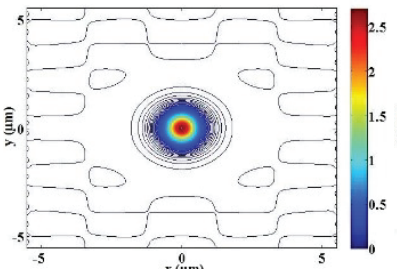

(a) Hy mode

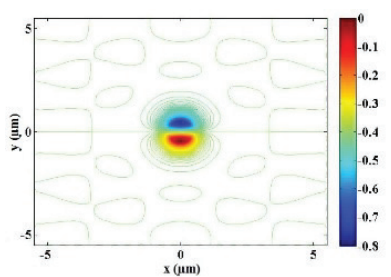

(c) $\mathrm{Hz}$ mode

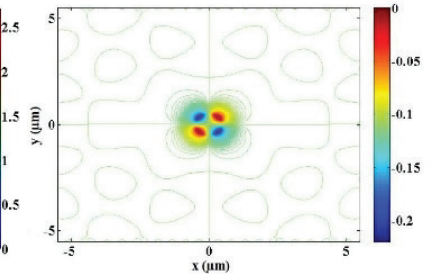

(b) Hx mode

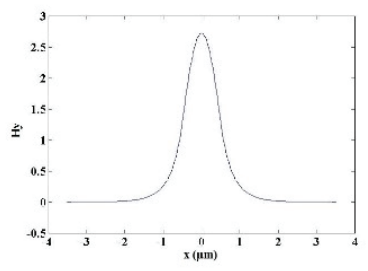

(d) 2d plot for Hy mode
Fig 1. Fundamental optical mode for $\mathrm{TF}$ with $\mathrm{R}=0.5 \mu \mathrm{m}$

Figure 2 (a), (b), and (c) show the fundamental Hy, Hx and $\mathrm{Hz}$ optical mode for GCTF with fiber radius equivalent to $0.5 \mu \mathrm{m}$ and clad with $0.1 \mu \mathrm{m}$ of graphene layer. Optical confinement is observed at the fiber surface, optic wave is trapped at the graphene layer due to it's higher refractive index [20]. Figure 2 (d) shows $2 d$ plot of Hy mode at -3.52 peak intensity. The strong confinement is contributed by the graphene layer.

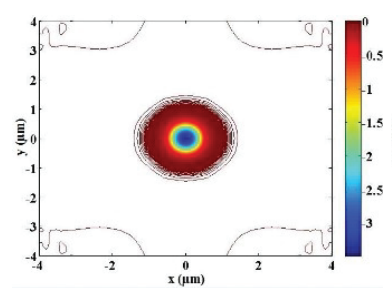

(a) Hy mode

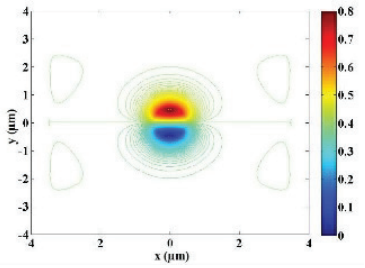

(c) $\mathrm{Hz}$ mode

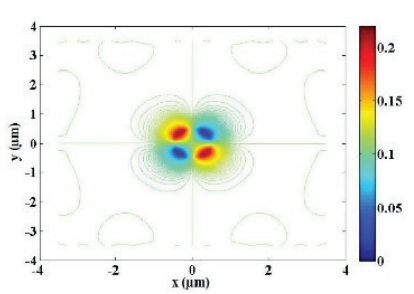

(b) Hx mode

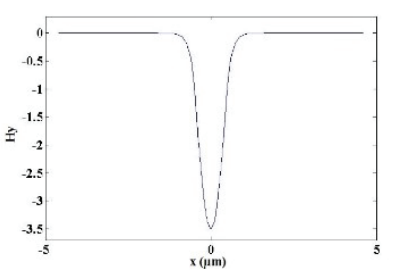

(d) $2 \mathrm{~d}$ plot for Hy mode
Fig 2. Fundamental optical mode for GCTF with $\mathrm{R}=0.5 \mu \mathrm{m}$.

\subsection{Acoustic wave}

Table 3 lists the acoustic wave output parameters. Based on Equation 3, $k_{\text {acoustic }}$ is solved by referring to the $\beta_{\text {optic }}$ result from Table 2 . GCTF has a higher acoustic velocity and higher acoustic frequency shift as compared to TF due to the high graphene velocity. Acoustic velocity of SAW for TF is recorded as $3306 \mathrm{~m} / \mathrm{s}$. Such result is in agreement with the SAW theory in which the velocity of propagation has to be less than the shear velocity $(3764 \mathrm{~m} / \mathrm{s})$ and longitudinal velocity $(5933 \mathrm{~m} / \mathrm{s})$. 
Table 3. Acoustic properties of TF and GCTF

\begin{tabular}{|c|c|c|}
\hline Properties & TF & GCTF \\
\hline$k_{\text {acoustic }}\left(\mu \mathrm{m}^{-1}\right)$ & 9.632 & 16.416 \\
\hline$v_{B}(\mathrm{GHz})$ & 5.237 & 10.198 \\
\hline$V_{a c}(\mathrm{GHz})$ & 3306 & 3822 \\
\hline
\end{tabular}

Significant difference can be observed in the acoustic result of TF and SMTF. SAW propagates on the fiber surface for TF, whereas acoustic confinement in the core of GCTF. Figure 3 (a), (b), and (c) show the Uz, Ux and Uy acoustic mode for TF. Figure 3 (d) verifies the result where two sharp peaks occur at 0.5 and -0.5 which indicate the fiber surface.

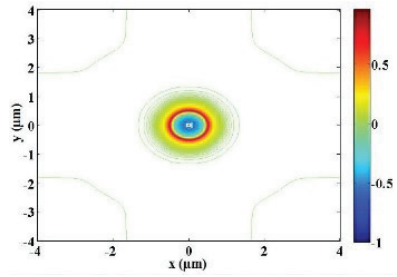

(a) Uz mode

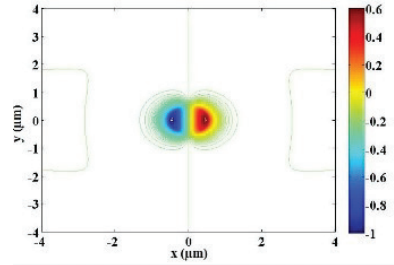

(c) Uy mode

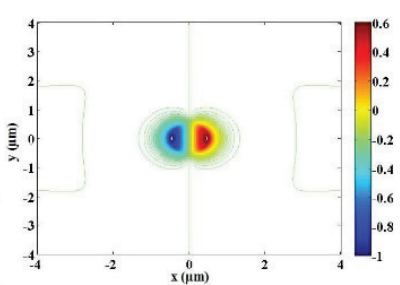

(b) Ux mode

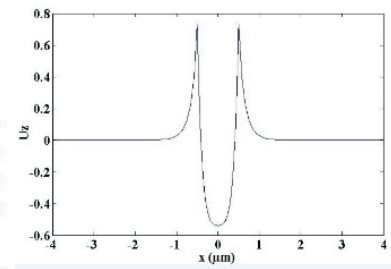

(d) $2 \mathrm{~d}$ plot for Uz mode
Fig 3. Fundamental acoustic mode for $\mathrm{TF}$ with $\mathrm{R}=0.5 \mu \mathrm{m}$

$0.1 \mu \mathrm{m}$ of graphene is then clad around TF, the acoustic wave confines from surface acoustic wave to hybrid acoustic wave (HAW). This can be explained in Equation 6 in where clad velocity has to be higher than core velocity to secure propagation of HAW. Figure 4 (d) confirms the mode propagation of HAW for tapered fiber clad with graphene layer.

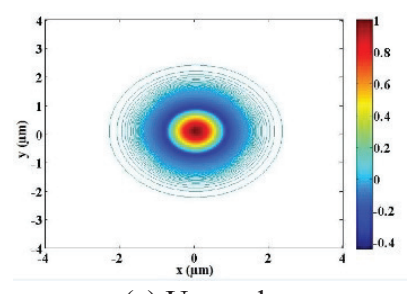

(a) Uz mode

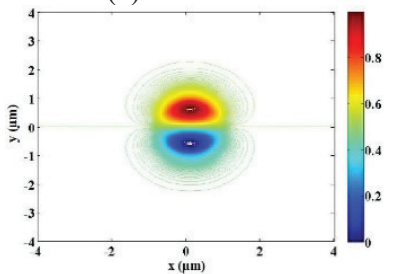

(c) Uy mode

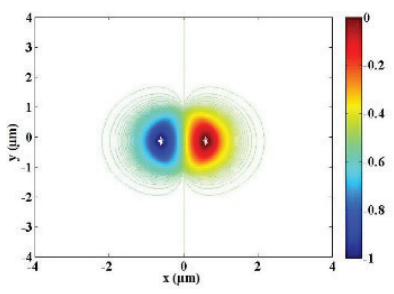

(b) Ux mode

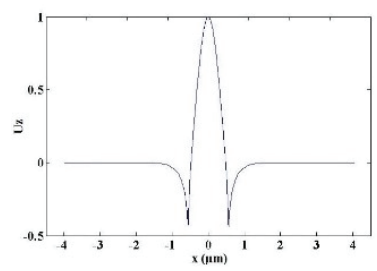

(d) 2 d plot for Uz mode
Fig 4. Fundamental acoustic mode for GCTF with $\mathrm{R}=0.5 \mu \mathrm{m}$

\subsection{Brillouin gain and threshold power}

In this subsection, the Brillouin gain and threshold power of TF and GCTF are calculated and analyzed. Table 4 lists the Brillouin gain and threshold power of TF and GCTF, calculation is performed based on Equation 9 and 10. Graphene cladding confines the acoustic mode propagation hence reduces the threshold power from $61.97 \mathrm{~W}$ down to $2.17 \mathrm{~W}$.

Table 4. Brillouin gain and threshold power of TF and GCTF

\begin{tabular}{|c|c|c|}
\hline Properties & TF & GCTF \\
\hline$v_{B}(\mathrm{GHz})$ & 5.237 & 10.198 \\
\hline$\Delta v_{B}(\mathrm{GHz})$ & 20 & 19 \\
\hline$g_{B}(\mathrm{~m} / \mathrm{W})$ & $1.419 \times 10^{-12}$ & $3.909 \times 10^{-11}$ \\
\hline$A_{\text {eff }}\left(\mathrm{m}^{2}\right)$ & $1.788 \times 10^{-13}$ & $1.732 \times 10^{-13}$ \\
\hline$P_{\text {th }}(\mathrm{W})$ & 61.87 & 2.17 \\
\hline
\end{tabular}

Graphene is a unique element with outstanding technical and mechanical properties. However, graphene properties used in this research are pre-defined to be in an ideal situation. In real time application, graphene properties might vary based on the preparatory method. Assuming an ideal case, graphene contribution in Stimulated Brillouin Scattering characterization is highlighted. Results demonstrate that graphene enhances the Brillouin gain therefore reduces the threshold power for tapered fiber. Such contribution would be interesting to be further explored in the real time experiment.

\section{Conclusion}

This research work was undertaken to perform Stimulated Brillouin Scattering characterization on the crosssectional region of tapered fiber and the change in result when graphene is clad on the fiber surface. One significant finding to emerge from this research work is that graphene layer lower the threshold power of a tapered fiber from $61.87 \mathrm{~W}$ down to $2.17 \mathrm{~W}$. Such result highlights that tapered fiber and graphene-clad tapered fiber with the gap in threshold power are applicable in the optical sensing and laser/amplifiers, respectively. It is recommended that experimental work can be done to identify the Brillouin scattering across graphene-clad tapered fiber to have a better understanding of graphene properties in SBS.

We thank Professor Azizur Rahman from City University London, United Kingdom, Dr and Dr. Siamak Emami from Shahid Baheshti University, Iran for the advice. Also, we thanks Universiti Tenaga Nasional internal grant RJO10289176 for the support. 


\section{References}

1. Brillouin, Léon. Ann. Phys.(Paris) 17, no. 88-122,21 (1922)

2. Keiser, Gerd. Optical fiber communications. John Wiley \& Sons, Inc. (2003)

3. Agrawal, Govind P. Nonlinear fiber optics. Academic press (2007)

4. Hicks, John W. U.S. Patent 4,778,238, (October 18, 1988)

5. Monro, Tanya M., Yvonne D. West, Daniel W. Hewak, N. G. R. Broderick, and D. J. Richardson. Elec. Lett. 36, no. 24: 1998-2000 (2000)

6. Woodward, R. I., E. J. R. Kelleher, S. V. Popov, and J. R. Taylor. Opt. Lett. 39, no. 8: 2330-2333 (2014)

7. Cherif, Rim, A. B. Salem, T. S. Saini, A. Kumar, Ravindra K. Sinha, and M. Zghal. Opt. Eng. 54, no. 7 (2015)

8. Hu, Kai, Irina V. Kabakova, Thomas FS Büttner, Simon Lefrancois, Darren D. Hudson, Sailing He, and Benjamin J. Eggleton. Opt. Lett. 39, no. 16: 4651-4654 (2014)

9. Beugnot, J.-Charles, S. Lebrun, G. Pauliat, H. Maillotte, V. Laude, and T. Sylvestre. Nat. com. 5 (2014)

10. M-Hernández, Carlos, D. Monzón-Hernández, I. Hernández-Romano, and J. Villatoro. Opt. exp. 23, no. 17 x: 22141-22148 (2014)

11. Kerttula, Juho, V. Filippov, V. Ustimchik, Y. Chamorovskiy, and Oleg G. Okhotnikov. Opt. exp. 20, no. 23: 25461-25470 (2012)

12. Harun, S. W., K. S. Lim, C. K. Tio, K. Dimyati, and H. Ahmad. Opt-Int J. for L. E. Opt. 124, no. 6: 538-543 (2013)

13. Hartung, A., F. Wirth, and H. Bartelt. In Proceedings to Progress in Electromagnetics Research Symposium, pp. 255-258 (2011)

14. Yadav, T. K., Narayanaswamy, R., Bakar, M. A., Kamil, Y. M., \& Mahdi, M. A. Opt. exp., 22(19), 22802-22807 (2014)

15. Layeghi, A., H. Latifi, and O. Frazão. 23rd International Conference on Optical Fiber Sensors, pp. 91570Z-91570Z (2014)

16. González-Cano, A., Navarrete, M.C., Esteban, Ó. and Díaz-Herrera, N., Sen. J., 14(3), pp.4791-4805 (2014)

17. P. Wang, G. Brambilla, M. Ding, Y. Semenova, Q. Wu, G. Farrell,J. Opt. Soc. Am. B 28 (5) 1180-1186 (2011)

18. Ascorbe, J., Corres, J.M., Arregui, F.J. and Matias, I.R., Sen. J., pp. 1916-1919 (2014)

19. E. W. Hill, A. Vijayaragahvan, K. Novoselov, Sen. J., IEEE 11 (12) 3161-3170 (2011)

20. B. Yao, Y. Wu, Q. Feng, Z. Wang, Y. Rao, Y. Chen, K. S. Chiang, Opt. Soc. A. pp. 4-6 (2015)

21. M. Batumalay, S. W. Harun, F. Ahmad, R. Md Nor, N. R. Zulkepely, H. Ahmad, Sens. J., IEEE 14 (5) 1704-1709 (2014)

22. H. Qiu, S. Xu, S. Jiang, Z. Li, P. Chen, S. Gao, C. Zhang, D. Feng, App. Surf. Sc. 329 390-395 (2015)

23. A. Shabaneh, S. Girei, P. Arasu, W. Rahman, A. Bakar, A. Sadek, H. Lim, N. Huang, M. Yaacob, Opt. Com. 331320 $324(2014)$

24. B. Yu, Y. Huang, M. Ding, Y. Ran, B. O. Guan, Fifth Asia Pacific Optical Sensors Conference, International Society for Optics and Photonics, pp. 1-4 (2015)

25. A. Gorbach, A. Marini, D. Skryabin, Opt. let. 38 (24), 5244-5247 (2013)

26. E. Madenci, I. Guven, Springer, (2015)

27. Y. Zhang, C. Yu, X. Fu, D. Li, W. Jia, W. Bi, Measurement 51 310-314 (2014)

28. J. A. Dehkordi, S. S. Hosseini, P. K. Kundu, N. R. Tan, Chem. Prod. \& Proc. Mod. 11 (1) 11-15 (2016)

29. P. Wriggers, J. Schröder, F. Auricchio, Mod. \& Sim. in Eng. Sc. 3 (1) 25 (2016)
30. M. Braginsky, C. Przybyla, Comp. Str. 136 538-545 (2016)

31. Y. S. Mamdem, X. Pheron, F. Taillade, Y. Jaoüen, R. Gabet, V. Lanticq, G. Moreau, A. Boukenter, Y. Ouerdane, S. Lesoille, et al.,Comsol conference (2010)

32. Z. Zhu, D. J. Gauthier, R. W. Boyd, Sc. (N.Y.) 318 (5857) 1748-1750 (2007)

33. M. R. Mokhtar, Ph.D. thesis, Univ. of Southampton (2005)

34. K. Virta, K. Mattsson, J. Sc.Com. 61 (1) 90$118(2014)$

35. C. G. Poulton, R. Pant, B. J. Eggleton, JOSA B 30 (10) 2657-2664 (2013)

36. S. Sriratanavaree, Ph.D. thesis, City University London (2014)

37. C. Lee, S. Chi, IEEE P. Tech. Lett. 12 (6) 672 $674(2000)$

38. Baimova, Y. A., Dmitriev, S. V., Savin. A. V., Kivshar, Y.S., Phy. of the Sol. State. 54 (4) 866-874 (2012) 